\title{
Invasive Fusariosis in Nonneutropenic Patients, Spain, 2000-2015
}

Elena Pérez-Nadales, Ana Alastruey-Izquierdo, María José Linares-Sicilia, Juan Carlos Soto-Debrán, Edson Abdala, Julio García-Rodríguez, Miguel Montejo, Patricia Muñoz, Miguel Salavert Lletí, Antonio Rezusta, Maite Ruiz Pérez de Pipaón, Lucrecia Yáñez, Esperanza Merino, María Isolina Campos-Herrero, José María Costa-Mateo, Jesús Fortún, Tomás García-Lozano, Carolina Garcia-Vidal, Mario Fernández-Ruiz, Ferrán Sánchez-Reus, Carmen Castro-Méndez, Inmaculada Guerrero-Lozano, Pere Soler-Palacín, José María Aguado, Luis Martínez-Martínez, Julian Torre-Cisneros, ${ }^{1}$ Marcio Nucci, ${ }^{1}$ the Spanish Fusariosis Study Group ${ }^{2}$

\section{Medscape ACTIVITY}

In support of improving patient care, this activity has been planned and implemented by Medscape, LLC and Emerging Infectious Diseases. Medscape, LLC is jointly accredited by the Accreditation Council for Continuing Medical Education (ACCME), the Accreditation Council for Pharmacy Education (ACPE), and the American Nurses Credentialing Center (ANCC), to provide continuing education for the healthcare team.

Medscape, LLC designates this Journal-based CME activity for a maximum of 1.00 AMA PRA Category 1 Credit(s) ${ }^{\mathrm{TM}}$. Physicians should claim only the credit commensurate with the extent of their participation in the activity.

Successful completion of this CME activity, which includes participation in the evaluation component, enables the participant to earn up to $1.0 \mathrm{MOC}$ points in the American Board of Internal Medicine's (ABIM) Maintenance of Certification (MOC) program. Participants will earn MOC points equivalent to the amount of CME credits claimed for the activity. It is the CME activity provider's responsibility to submit participant completion information to ACCME for the purpose of granting ABIM MOC credit.

All other clinicians completing this activity will be issued a certificate of participation. To participate in this journal CME activity: (1) review the learning objectives and author disclosures; (2) study the education content; (3) take the post-test with a $75 \%$ minimum passing score and complete the evaluation at http://www.medscape.org/journal/eid; and (4) view/print certificate. For CME questions, see page 339.

Release date: December 22, 2020; Expiration date: December 22, 2021

Learning Objectives

Upon completion of this activity, participants will be able to:

- Describe the incidence of invasive fusariosis (IF) and fungal isolates from patients with IF in 18 Spanish hospitals during the 15year period from 2000 to 2015 , according to a retrospective observational study

- Determine the clinical features of patients with IF in 18 Spanish hospitals during the 15-year period from 2000 to 2015 according to a retrospective observational study

- Identify the treatment and outcomes of patients with IF in 18 Spanish hospitals during the 15-year period from 2000 to 2015 , according to a retrospective observational study.

CME Editor

Deanna Altomara, BA, Copyeditor, Emerging Infectious Diseases. Disclosure: Deanna Altomara, BA, has disclosed no relevant financial relationships.

CME Author

Laurie Barclay, MD, freelance writer and reviewer, Medscape, LLC. Disclosure: Laurie Barclay, MD, has disclosed no relevant financial relationships.

Authors

Disclosures: Elena Pérez-Nadales, PhD; María José Linares-Sicilia, PhD; Juan Carlos Soto-Debrán, MS; Edson Abdala, PhD; Miguel Montejo, MD; Antonio Rezusta, PhD; Maite Ruiz Pérez de Pipaón, PhD; Lucrecia Yáñez, MD, PhD; Esperanza Merino, MD; María Isolina Campos-Herrero, PhD; José María Costa-Mateo, MD; Jesús Fortún, PhD; Tomás García-Lozano, MD; Mario Fernández-Ruiz, PhD; Ferrán Sánchez-Reus, PhD; Carmen CastroMéndez, PhD; Inmaculada Guerrero-Lozano, PhD; Pere Soler-Palacín, MD, PhD, MSc; José María Aguado, PhD; and Luis Martínez-Martínez, MD, PhD, have disclosed no relevant financial relationships. Ana Alastruey-lzquierdo, PhD, has disclosed the following relevant financial relationships: served as a speaker or a member of a speakers bureau for Gilead Sciences, Inc.; Merck Sharp \& Dohme GmbH; Pfizer Inc.; received grants for clinical research from F2G Ltd. Julio García-Rodríguez, PhD, has disclosed the following relevant financial relationships: served as a speaker or a member of a speakers bureau for Gilead Sciences. Inc.; Pfizer Inc. Patricia Muñoz, MD, PhD, has disclosed the following relevant financial relationships: served as an advisor or consultant for Astellas Pharma, Inc.; Basilea Pharmaceutica Ltd.; Gilead Sciences, Inc.; Merck Sharp \& Dohme GmbH; Nabriva Therapeutics plc; Pfizer Inc.; served as a speaker or a member of a speakers bureau for Angelini; Astellas Pharma, Inc.; Basilea Pharmaceutica Ltd.; Gilead Sciences, Inc.; Merck Sharp \& Dohme $\mathrm{GmbH}$; Pfizer Inc.; Roche; Werfen; received grants for clinical research from Astellas Pharma, Inc.; CIBERES; Fabbrica Italiana Sintetici S.P.A.; T2 Biosystems, Inc. Miguel Salavert Lletí, PhD, has disclosed the following relevant financial relationships: served as an advisor or consultant for: Gilead Sciences, Inc.; Merck Sharp \& Dohme GmbH; Pfizer Inc.; served as a speaker or a member of a speakers bureau for: Gilead Sciences, Inc.; Janssen-Cilag; Merck Sharp \& Dohme $\mathrm{GmbH}$; Pfizer Inc. Carolina Garcia-Vidal, PhD, has disclosed the following relevant financial relationships: served as a speaker or a member of a speakers bureau for Gilead Sciences, Inc.; Janssen-Cilag; Merck Sharp \& Dohme GmbH; Novartis Pharmaceuticals Corporation; Pfizer Inc.; received grants for clinical research from Gilead Sciences, Inc.; Merck Sharp \& Dohme GmbH. Julián Torre-Cisneros, MD, PhD, has disclosed the following relevant financial relationships: served as an advisor or consultant for Merck Sharp \& Dohme GmbH; Pfizer Inc.; Shionogi \& Co., Ltd.; served as a speaker or a member of a speakers bureau for: Merck Sharp \& Dohme GmbH; Pfizer Inc.; received grants for clinical research from Pfizer Inc. Marcio Nucci, MD, has disclosed the following relevant financial relationships: served as an advisor or consultant for Merck Sharp \& Dohme GmbH; served as a speaker or a member of a speakers bureau for AbbVie Inc.; Astellas Pharma, Inc.; Basilea Pharmaceutica Ltd.; Gilead Sciences, Inc.; Merck Sharp \& Dohme GmbH; Pfizer Inc. 
Invasive fusariosis (IF) is associated with severe neutropenia in patients with concurrent hematologic conditions. We conducted a retrospective observational study to characterize the epidemiology of IF in 18 Spanish hospitals during 2000-2015. In that time, the frequency of IF in nonneutropenic patients increased from 0.08 cases per 100,000 admissions in 2000-2009 to 0.22 cases per 100,000 admissions in 2010-2015. Nonneutropenic IF patients often had nonhematologic conditions, such as chronic cardiac or lung disease, rheumatoid arthritis, history of solid organ transplantation, or localized fusariosis. The 90-day death rate among nonneutropenic patients $(28.6 \%)$ and patients with resolved neutropenia (38.1\%) was similar. However, the death rate among patients with persistent neutropenia (91.3\%) was significantly higher. We used a multivariate Cox regression analysis to characterize risk factors for death: persistent neutropenia was the only risk factor for death, regardless of antifungal therapy.

Tnvasive fusariosis (IF) is a fungal disease that Imostly affects patients with hematologic malignancies or who have received hematopoietic cell transplants. These patients often have prolonged and profound neutropenia, low levels of T cells, or both $(1,2)$. Despite advances in early diagnosis and treatment, IF remains associated with high morbidity and death rates $(3,4)$.

Most studies on this fungal disease have occurred in North and South America (4-9). However, the epidemiology of IF in Europe has not been fully characterized; within Europe, most multicenter studies on IF have occurred in Italy and France $(8,10-12)$.

Author affiliations: Spanish Network for Research in Infectious Diseases, Instituto de Salud Carlos III, Madrid, Spain (E. PérezNadales, A. Alastruey-Izquierdo, M. Montejo, M. Ruiz Pérez de Pipaón, L. Yáñez, J. Fortún, C. Garcia-Vidal, M. Fernández-Ruiz, I. Guerrero-Lozano, P. Soler-Palacín, J.M. Aguado, L. MartínezMartínez, J. Torre-Cisneros); Maimonides Biomedical Research Institute of Cordoba, Reina Sofia University Hospital, University of Cordoba, Cordoba, Spain (E. Perez-Nadales, M.J. Linares Sicilia, J. M. Costa-Mateo, L. Martínez-Martínez, J. Torre-Cisneros); Centro Nacional de Microbiología, Instituto de Salud Carlos III, Madrid, Spain (A. Alastruey-Izquierdo, J.C. Soto-Debrán); Hospital das Clinicas, Universidade de São Paulo, São Paulo, Brazil (E. Abdala); Hospital Universitario La Paz, Madrid (J. García-Rodríguez); Hospital Universitario Cruces, Baracaldo, Spain (M. Montejo); Hospital General Universitario Gregorio Marañón, Instituto de Investigación Sanitaria Hospital Gregorio Marañón, Universidad Complutense de Madrid, Madrid, Spain (P. Muñoz); Hospital Universitario "12 de Octubre," Instituto de Investigación Hospital "12 de Octubre," Universidad Complutense, Madrid, Spain (M. Fernández-Ruiz, J.M. Aguado); Hospital
A multicenter study by the European Confederation of Medical Mycology reported 76 cases of IF during 2007-2012 (13). Most (60\%) of these IF cases occurred in Italy but none in Spain. Previous single-center studies in Europe have reported regional differences in the distribution of Fusarium species and their susceptibilities to antimicrobial drugs, highlighting the importance of monitoring local epidemiologic data (13-15). We characterized the epidemiology of IF in Spain using a retrospective observational study that examined the effects of clinical and etiologic characteristics on outcomes in a cohort of 58 IF patients at hospitals in Spain.

\section{Methods}

We conducted this study in 18 hospitals in Spain, 8 of which belong to the Spanish Network for Research in Infectious Diseases, Instituto de Salud Carlos III, in Madrid, Spain. The study was approved by Reina Sofía University Hospital Institutional Review Board (Córdoba, Spain), which waived the need to obtain written informed consent. During January 2000-December 2015, hospital staff reviewed microbiological and pathologic registries to identify cases of IF. Data were recorded in a password-protected, electronic clinical research file. We monitored the collected data for missing information, inconsistencies, and ambiguities; when necessary, we sent queries to the appropriate hospitals for clarification.

We conducted a blind review of reported cases of IF. In this study, we included only proven cases of IF according to the consensus definitions of invasive fungal diseases established by the European

Universitario y Politécnico La Fe, Valencia, Spain (M. Salavert Lletí); Hospital Universitario Miguel Servet, Universidad de Zaragoza, Instituto de Investigación Sanitaria Aragón, Zaragoza, Spain (A. Rezusta); Hospital Universitario Virgen del Rocío, Seville, Spain (M. Ruiz Pérez de Pipaón); Hospital Universitario Marqués de Valdecilla, Instituto de Investigación Sanitaria Valdecilla, Santander, Spain (L. Yáñez); Hospital General Universitario de Alicante, Spain (E. Merino); Hospital Universitario de Gran Canaria Doctor Negrín, Las Palmas de Gran Canaria, Spain (M. I. Campos-Herrero); Hospital Ramón y Cajal, Madrid (J. Fortún); Fundación Instituto Valenciano de Oncología, Valencia, Spain (T. García-Lozano); Hospital Universitario de Bellvitge, Barcelona, Spain (C. Garcia-Vidal); Hospital de la Santa Creu i Sant Pau, Barcelona (F. Sánchez-Reus); Hospital Universitario Virgen de Valme, Seville (C. Castro-Méndez); Hospital Universitario Puerta del Mar, Cádiz, Spain (I. GuerreroLozano); Hospital Universitari Vall d'Hebron, Barcelona (P. Soler-Palacín); Universidade Federal do Rio de Janeiro, Rio de Janeiro, Brazil (M. Nucci)

DOI: https://doi.org/10.3201/eid2701.190782 
Organization for Research and Treatment of Cancer and the Mycoses Study Group Education and Research Consortium $(16,17)$. IF can be proven in 4 ways: microscopic examination of a specimen obtained by needle aspiration or biopsy that documents hyphae and isolates Fusarium spp. in the same tissue; blood culture yielding Fusarium spp. alongside signs consistent with an infectious disease process; isolation of Fusarium spp. in a normally sterile site (excluding bronchoalveolar lavage fluid, a paranasal or mastoid sinus cavity specimen, and urine) with accompanying signs of infection; or amplification and sequencing of Fusarium DNA in formalinfixed paraffin-embedded tissue $(16,17)$.

The participating hospitals identified Fusarium isolates using genetic sequencing, matrix-assisted laser desorption/ionization time-of-flight (MALDITOF) mass spectrometry, or morphologic characteristics. In addition, all biological samples available at the time of this retrospective study (i.e., Fusarium isolates and specimens for biopsies) were sent to the Mycology Reference Laboratory at the Instituto de Salud Carlos III for confirmatory genetic sequencing. The isolates were freeze-dried on potato agar slants on arrival and stored in distilled water. Molecular identification was based on the translation elongation factor 1a gene (18). Isolates were cultured in GYEP medium $(0.3 \%$ yeast extract, $1.0 \%$ peptone; Francisco Soria Melguizo S.A., http://www.f-soria.es) with $2.0 \%$ glucose (Sigma-Aldrich Inc., https://www.sigmaaldrich.com) at $30^{\circ} \mathrm{C}$ for $24-48 \mathrm{~h}$. Genomic DNA was isolated by using an extraction procedure described previously (19). Internal transcribed spacer region and a portion of the translation elongation factor 1a gene region were amplified as previously described (20). Alignment results were obtained and edited using Lasergene MegAlign Pro software from DNASTAR, Inc. (https://www.dnastar.com). All sequences were compared with reference sequences from GenBank and MycoBank (https://www.mycobank.org) databases by using InfoQuest FP version 4.50 software (Bio-Rad Laboratories, https://www. bio-rad.com). We also used an in-house database of the Mycology Reference Laboratory. When only biopsy specimens were available, we used real-time PCR specific for Fusarium species as previously described (21). When identified cases did not have available isolates or biologic material for PCR, we reported the specific Fusarium species only if obtained by MALDITOF mass spectrometry in conjunction with histopathology. We reported the remaining IF cases in patients with compatible clinical signs and symptoms as caused by Fusarium spp.
We defined survival as the time between the date of diagnosis and death or end of follow-up care (i.e., 90 days). We defined the date of diagnosis as the day of the first Fusarium-positive culture. We defined disseminated fusariosis as the involvement of $>1$ noncontiguous organ. We did not consider fungemia cases as disseminated disease unless $>1$ organ was involved (e.g., skin, lung, or sinuses) (3). We defined neutropenia as a blood neutrophil count $<500$ cells/ $\mathrm{mm}^{3}$ temporally related to the onset of fungal disease. We defined persistent neutropenia as cases with continued low neutrophil counts at the end of treatment or death. We defined resolution of neutropenia as a persistent recovery of blood neutrophil count $>500$ / $\mathrm{mm}^{3}$ as determined by available hospital records.

We calculated the incidence of IF using total admissions in the participating hospitals as the denominator; we expressed the incidence as the number of cases per 100,000 admissions. For statistical purposes, we arbitrarily defined 2 time periods: 2000-2009 and 2010-2015. We compared incidences between the different periods by $\chi^{2}$ test using WinPepi version 11.65 (Brixton Health, http://www.brixtonhealth.com). We considered $p \leq 0.05$ to be significant.

We collected variables describing patient demographic data, concurrent conditions, neutropenia, receipt of corticosteroids, clinical manifestations, diagnostic procedures, treatment, and outcome (i.e., 90-day survival). We compared categorical variables using $\chi^{2}$ or 2-tailed Fisher exact test, and we compared continuous variables using the Mann-Whitney U test. We conducted all statistical tests with SPSS Statistics 16.0 (IBM Inc., https://www.ibm.com) and $\mathrm{R}$ version 3.5.0 (R Foundation for Statistical Computing, https://www.rproject.org); we considered 2-sided $\mathrm{p}$ values $<0.05$ to be significant. We constructed unadjusted KaplanMeier curves and compared them using the log-rank test. In addition, we evaluated factors associated with 90-day survival. We evaluated the following variables by univariate and multivariable Cox regression analyses: age, sex, age-adjusted Charlson comorbidity index (22), underlying disease, receipt of antifungals and corticosteroids in the previous 30 days, neutropenia at diagnosis of fusariosis and at the end of follow-up, clinical manifestations, and primary treatment (monotherapy with a lipid formulation of amphotericin B, monotherapy with voriconazole, or combination treatment). We entered variables with $p<0.1$ by univariate analysis into the multivariate analysis; we included variables with $p<0.05$ by the multivariate analysis in the final model. We evaluated the prediction accuracy of the final Cox model using the area under the receiver operating characteristic curve. 


\section{Results}

\section{Incidence of IF}

We identified 75 patients with Fusarium spp. isolated from clinical samples at 18 hospitals in Spain (Appendix Table 1, https://wwwnc.cdc.gov/EID/ article/27/1/19-0782-App1.pdf) during 2000-2015. We excluded 10 patients with superficial infections and 7 with probable cases. In this study, the overall incidence of IF during 2000-2015 was 0.55 cases/100,000 admissions, corresponding to 0.42 neutropenic and 0.13 nonneutropenic patients $/ 100,000$ admissions $(p<0.01)$. The overall incidence of IF was 0.40 cases/100,000 admissions during 2000-2009 and 0.79 cases/100,000 admissions during 2010-2015 ( $\mathrm{p}<0.01$ ). Among neutropenic patients, the incidence of IF increased from 0.32 to 0.57 cases $/ 100,000$ admissions $(p=0.06)$. Among nonneutropenic patients, the incidence of IF increased from 0.08 to 0.22 cases $/ 100,000$ admissions $(p=0.05)$. We also determined the annual cumulative incidence curves for the 2 groups of patients (Appendix Figure 1).

\section{Clinical Characteristics}

We identified 58 cases of IF: $44(75.9 \%)$ occurred in neutropenic patients and $14(24.1 \%)$ in nonneutropenic patients (Table 1). Most (59\%) patients were male. The median age was 67 years (interquartile range [IQR] 38-19 years) in neutropenic patients and 45 years (IQR 28-65 years) in nonneutropenic patients $(p=0.05)$. In total, $36.4 \%$ of neutropenic and $42.9 \%$ of nonneutropenic patients had received corticosteroid therapy in the previous 30 days $(p=0.66) ; 72.7 \%$ of neutropenic and $35.7 \%$ of nonneutropenic patients had received antifungal therapy in the previous 30 days $(p=0.66)$. At the end of follow-up (i.e., 90 days after diagnosis), 23 (52.3\%) neutropenic patients had persistent neutropenia.

In total, 41 (93.2\%) neutropenic and $5(35.7 \%)$ nonneutropenic patients had hematologic malignancies $(p<0.01)$. In the neutropenic patient group, the most common hematologic malignancies were acute myeloid leukemia (43.2\%) and acute lymphoid leukemia $(18.2 \%)$. The proportion of patients who had received hematopoietic stem cell transplants (HSCTs) was 25\% (11/44; 8 allogeneic, 2 autologous, and 1 cord blood haploidentical) in neutropenic patients versus $21.4 \%$ $(3 / 14 ; 1$ allogeneic and 2 autologous) in nonneutropenic patients $(p=1.00)$. Four $(9.7 \%)$ neutropenic patients and $1(7.1 \%)$ nonneutropenic patient had graft-versushost disease $(p=1.00)$. Overall, $21.4 \%$ of nonneutropenic patients and $2.3 \%$ of neutropenic patients had a history of solid organ transplantation $(p=0.04)$. Finally,
$35.7 \%$ of nonneutropenic and $2.3 \%$ of neutropenic patients had other underlying conditions $(p=0.04)$. Five nonneutropenic patients had chronic cardiac disease, T-cell prolymphocytic leukemia, rheumatoid arthritis, chronic obstructive pulmonary disease, or infantile respiratory distress syndrome; 1 neutropenic patient had hemophagocytic lymphohistiocytosis.

Neutropenic patients were more likely than nonneutropenic patients to have a fever at the time of diagnosis $(90.9 \%$ vs. $57.1 \%$; $p<0.01)$. Neutropenic patients also were more likely to have skin lesions $(65.9 \% \mathrm{vs}$. $21.4 \% ; \mathrm{p}<0.01)$. We did not observe a difference in the proportion of patients with lung involvement in the IF infection $(72.7 \%$ of neutropenic vs. $64.3 \%$ of nonneutropenic patients; $\mathrm{p}=0.74$ ). We also did not observe a difference in the rate of concurrent infections (56.8\% of neutropenic vs. $42.9 \%$ of nonneutropenic patients; $p$ $=0.36$ ) (Appendix Table 2). Disseminated disease occurred more frequently among neutropenic patients $(79.5 \%)$ than nonneutropenic patients $(28.6 \% ; \mathrm{p}<0.01)$. In contrast, localized forms of IF, especially localized pneumonia $(6.8 \%$ of neutropenic patients vs. $35.7 \%$ of nonneutropenic patients; $p=0.02)$ and fungemia $(6.8 \%$ of neutropenic patients vs. $28.6 \%$ of nonneutropenic patients; $p=0.05$ ), were more common among nonneutropenic patients. However, disseminated fungemia was more common among neutropenic than nonneutropenic patients $(45.7 \%$ vs. $25 \%$; $p=0.03)$. Among neutropenic patients, skin lesions were more common in persons with disseminated IF (77.1\%) than localized IF $(22.2 \% ; p=0.004)$; lung involvement also was more common among those with disseminated IF ( $82.9 \%$ vs. $33.3 \% ; p=0.007)$.

Nonneutropenic patients were more likely than neutropenic patients to have a diagnosis on the basis of positive culture alone ( $43.2 \%$ of neutropenic patients vs. $85.7 \%$ of nonneutropenic patients; $p<0.01$ ). Neutropenic patients were more likely to have a diagnosis on the basis of a positive culture and histopathology $(52.3 \%)$ than nonneutropenic patients $(14.3 \%$; $\mathrm{p}=0.01)$.

\section{Isolated Fusarium Species}

Thirty-six patients $(62.1 \%)$ received a species-level etiologic diagnosis using molecular methods: 26 (44.8\%) by genetic sequencing and $10(17.2 \%)$ by MALDI-TOF mass spectrometry (Table 2). Among these patients, F. solani species complex (SC) was the most common $(18 / 58 ; 31.0 \%)$, along with Gibberella fujikuroi SC (10/58; 17.2\%), and F. oxysporum SC (5/58; 8.6\%). The remaining $22(37.9 \%)$ cases were identified as caused by Fusarium spp. Among disseminated infections, most $(74.4 \%)$ were reported to species level; the most 
common were F. solani SC (15/39; 38.5\%), F. fukikuroi SC (8/39; 20.5\%), and F. oxysporum SC (3/39; 7.7\%). In the cohort, disseminated IF also was caused by $2 \mathrm{~F}$. brachygibbosum infections and $1 \mathrm{~F}$. dimerum SC infection. Most (63.2\%) localized infections were caused by Fusarium spp. F. solani SC (2/39; 5.1\%), F. fukikuroi SC
$(2 / 39 ; 5.1 \%)$, and F. oxysporum SC (3/39; 7.7\%) were the most common etiologic agents.

\section{Therapeutic Regimens}

Overall, $62.1 \%$ of patients received monotherapy $(29.3 \%$ azoles, $32.8 \%$ amphotericin B), $27.6 \%$ of

\begin{tabular}{|c|c|c|c|c|}
\hline Variables & Total & Nonneutropenic & Neutropenic & $\mathrm{p}$ valuet \\
\hline Total & $58(100.0)$ & $14(100.0)$ & $44(100.0)$ & \\
\hline \multicolumn{5}{|l|}{ Sex } \\
\hline M & $34(58.6)$ & $8(57.1)$ & $26(59.1)$ & 0.9 \\
\hline $\mathrm{F}$ & $24(41.4)$ & $6(42.9)$ & $18(40.9)$ & 0.9 \\
\hline Median age, y (IQR) & $51(31-67)$ & $67(38-79)$ & $45(28-65)$ & $0.05 \ddagger$ \\
\hline \multicolumn{5}{|l|}{ Treatment history (previous $30 \mathrm{~d}$ ) } \\
\hline Antifungal & $37(63.8)$ & $5(35.7)$ & $32(72.7)$ & 0.01 \\
\hline Corticosteroid & $22(37.9)$ & $6(42.9)$ & $16(36.4)$ & 0.66 \\
\hline Persistent neutropenia & $23(39.7)$ & 0 & $23(52.3)$ & \\
\hline \multicolumn{5}{|l|}{ Concurrent conditions } \\
\hline Hematologic malignancy & $46(79.3)$ & $5(35.7)$ & $41(93.2)$ & $<0.01 \S$ \\
\hline Acute myeloid leukemia & $20(34.5)$ & $1(7.1)$ & $19(43.2)$ & \\
\hline Acute lymphoid leukemia & $8(13.8)$ & 0 & $8(18.2)$ & \\
\hline Non-Hodgkin Iymphoma & $5(8.6)$ & $2(14.3)$ & $3(6.8)$ & \\
\hline Aplastic anemia & $4(6.9)$ & 0 & $4(9.1)$ & \\
\hline Multiple myeloma & $3(5.2)$ & $2(14.3)$ & $1(2.3)$ & \\
\hline Myelodysplasia & $2(3.4)$ & 0 & $2(4.5)$ & \\
\hline Chronic lymphoid leukemia & $2(3.4)$ & 0 & $2(4.5)$ & \\
\hline Chronic myeloid leukemia & $1(1.7)$ & 0 & $1(2.3)$ & \\
\hline Hodgkin's lymphoma & $1(1.7)$ & 0 & $1(2.3)$ & \\
\hline History of hematopoietic stem cell transplant & $14(24.1)$ & $3(21.4)$ & $11(25.0)$ & $1.00 \S$ \\
\hline Allogenic & $9(15.5)$ & $1(7.1)$ & $8(18.2)$ & \\
\hline Autologous & $4(6.9)$ & $2(14.3)$ & $2(4.5)$ & \\
\hline Cord blood haploidentical & $1(1.7)$ & 0 & $1(2.3)$ & \\
\hline Graft-versus-host disease & $5(8.6)$ & $1(7.1)$ & $4(9.1)$ & $1.00 \S$ \\
\hline Acute & $3(5.2)$ & 0 & $3(6.8)$ & \\
\hline Chronic & $2(3.4)$ & $1(7.1)$ & $1(2.3)$ & \\
\hline Solid tumor & $2(3.4)$ & $1(7.1)$ & $1(2.3)$ & $0.43 \S$ \\
\hline History of solid organ transplant & $4(6.9)$ & $3(21.4)$ & $1(2.3)$ & $0.04 \S$ \\
\hline Otherף & $10(17.2)$ & $5(35.7)$ & $1(2.3)$ & $<0.01 \S$ \\
\hline \multicolumn{5}{|l|}{ Clinical manifestations } \\
\hline Fever & $48(82.8)$ & $8(57.1)$ & $40(90.9)$ & $<0.01 \S$ \\
\hline Skin lesions & $32(55.2)$ & $3(21.4)$ & $29(65.9)$ & $<0.01$ \\
\hline Lung involvement & $41(70.7)$ & $9(64.3)$ & $32(72.7)$ & $0.74 \S$ \\
\hline Sinusitis & $9(15.5)$ & $1(7.1)$ & $8(18.2)$ & $0.43 \S$ \\
\hline Blindness & $4(6.9)$ & 0 & $4(9.1)$ & $0.56 \S$ \\
\hline Concurrent infection & $31(53.4)$ & $6(42.9)$ & $25(56.8)$ & 0.36 \\
\hline Bacterial & $17(29.3)$ & $2(14.3)$ & $15(34.1)$ & $0.18 \S$ \\
\hline Fungal & $4(6.9)$ & $1(7.1)$ & $4(9.1)$ & $0.56 \S$ \\
\hline Viral & $6(10.3)$ & $3(21.4)$ & $3(6.8)$ & $0.15 \S$ \\
\hline Polymicrobial & $4(6.9)$ & $1(7.1)$ & $3(6.8)$ & $1.00 \S$ \\
\hline \multicolumn{5}{|l|}{ Type of fusariosis } \\
\hline Localized & $19(32.8)$ & $10(71.4)$ & $9(20.5)$ & $<0.01 \S$ \\
\hline Cutaneous, localized & $3(5.2)$ & $1(7.1)$ & $2(4.5)$ & $1.00 \S$ \\
\hline Pneumonia & $8(13.8)$ & $5(35.7)$ & $3(6.8)$ & $0.02 \S$ \\
\hline Sinusitis & $1(1.7)$ & $\begin{array}{c}0 \\
0\end{array}$ & $1(2.3)$ & $1.00 \S$ \\
\hline Fungemia & $7(12.1)$ & $4(28.6)$ & $3(6.8)$ & $0.05 \S$ \\
\hline Disseminated & $39(67.2)$ & $4(28.6)$ & $35(79.5)$ & $<0.01 \S$ \\
\hline \multicolumn{5}{|l|}{ Diagnosis } \\
\hline Culture & $31(53.4)$ & $12(85.7)$ & $19(43.2)$ & $<0.01$ \\
\hline Culture and histopathology & $25(43.1)$ & $2(14.3)$ & $23(52.3)$ & 0.01 \\
\hline Histopathology & $2(3.4)$ & 0 & $2(4.5)$ & 1.00 \\
\hline
\end{tabular}

*Values are no. (\%), except where indicated.

tp values obtained by $\chi^{2}$ test, except where indicated.

fDetermined by Mann-Whitney U test.

$\S$ Determined by Fisher exact test.

TOther conditions reported include hemophagocytic lymphohistiocytosis, chronic cardiac disease, T-cell prolymphocytic leukemia, rheumatoid arthritis

chronic obstructive pulmonary disease, and infantile respiratory distress syndrome. 
Table 2. Distribution of isolated Fusarium species, Spain, 2000-2015*

\begin{tabular}{|c|c|c|c|c|}
\hline Species & Total & Localized & Disseminated & $\mathrm{p}$ value \\
\hline Total & $58(100.0)$ & $19(100.0)$ & $39(100.0)$ & \\
\hline F. solani SC & $18(31.0)$ & $3(15.8)$ & $15(38.5)$ & $0.08 \ddagger$ \\
\hline Gibberella fujikuroi SC & $10(17.2)$ & $2(10.5)$ & $8(20.5)$ & 0.29 \\
\hline F. proliferatum & $6(10.3)$ & 0 & $6(15.4)$ & 0.08 \\
\hline F. verticillioides & $3(5.2)$ & $2(10.5)$ & $1(2.6)$ & 0.25 \\
\hline F. fujikuroi & $1(1.7)$ & 0 & $1(2.6)$ & 0.67 \\
\hline F. oxysporum SC & $5(8.6)$ & $2(10.5)$ & $3(7.7)$ & 0.53 \\
\hline F. brachygibbosum & $2(3.4)$ & 0 & $2(5.1)$ & 0.45 \\
\hline F. dimerum SC & $1(1.7)$ & 0 & $1(2.6)$ & 0.67 \\
\hline Fusarium spp. & $22(37.9)$ & $12(63.2)$ & $10(25.6)$ & $<0.01$ \\
\hline
\end{tabular}

patients received combination therapy (e.g., azoles plus amphotericin B, mostly liposomal amphotericin B plus voriconazole), and $10.4 \%$ of patients did not receive an active treatment ( 3 patients were not treated, 2 received empirical caspofungin, and 1 received empirical micafungin) (Appendix Table 3). Nonneutropenic patients were more likely than neutropenic patients to receive voriconazole monotherapy ( $54.5 \%$ vs. $85.7 \% ; \mathrm{p}<0.01)$. We did not observe a difference in the proportions of patients who were treated with amphotericin B (36.4\% of neutropenic patients vs. $21.4 \%$ of nonneutropenic patients; $\mathrm{p}=0.35)$. Sixteen $(36.4 \%)$ neutropenic patients received combination therapy: 14 received a lipid formulation of amphotericin $B$ plus voriconazole and 2 received amphotericin B plus posoconazole. No nonneutropenic patients received combination therapy.

\section{Outcomes}

By 90 days after diagnosis, $56.9 \%$ of all patients had died: $28.6 \%$ of nonneutropenic patients and $65.9 \%$ of patients with neutropenia at IF onset $(p=0.01)$. We analyzed these results using unadjusted KaplanMeier survival graphs (Appendix Figure 2; $p=0.03$ by log-rank test). The death rate of nonneutropenic patients $(28.6 \%)$ was similar to that of patients who had recovered from neutropenia $(38.1 \% ; \mathrm{p}=0.56)$ and both rates were significantly lower than among patients with persistent neutropenia $(91.3 \%$; $<<0.01)$.

After 90 days, $66.7 \%$ of patients with localized and $36.8 \%$ with disseminated disease had died $(\mathrm{p}=$ $0.03)$. Overall, $66.7 \%(2 / 3)$ of patients with localized disease of the skin/soft tissue, $50 \%$ (4/8) with lung involvement, and $14.3 \%(1 / 7)$ with fungemia died.

In the assessment of prognostic factors, we analyzed $50 \mathrm{IF}$ patients who received active antifungal therapy. In univariate analysis, 90-day death risk was associated with neutropenia at onset of IF and persistent neutropenia. Age, sex, age-adjusted Charlson comorbidity index, corticosteroid therapy in the previous
30 days, administration of antifungal in the previous 30 days, hematologic malignancy, history of HSCT, fungemia, disseminated disease, and the treatment type (i.e., monotherapy with a lipid formulation of amphotericin B, monotherapy with azoles or combined therapy) were not associated with 90-day survival. In multivariate analysis, persistent neutropenia (hazard ratio [HR] 7.08, 95\% CI 1.91-26.17; p<0.01) was significantly associated with 90-day death risk (Table 3). We calculated adjusted Kaplan-Meier survival curves for the Cox regression model (Figure; area under the receiver operating characteristic curve $=0.83$ ).

\section{Discussion}

We described 58 cases of invasive Fusarium infections at 18 hospitals in Spain during a 15-year period (2000-2015). During this time, IF incidence increased from 0.40 (2000-2009) to 0.79 (2010-2015) cases per 100.000 admissions $(p<0.01)$. Incidence of IF in neutropenic patients increased from 0.32 to 0.57 cases per 100,000 admissions ( $p=0.06)$. We observed a 3 -fold increase in the incidence of IF in nonneutropenic patients, from 0.08 to 0.22 cases per 100,000 admissions $(p=0.05)$. This increase might have been caused by an increase in the at-risk population, environmental exposure to Fusarium conidia, the increased use of antifungal prophylaxis, or a combination of these factors.

To date, the highest IF incidence rates worldwide are in healthcare centers in Brazil. A cohort of HSCT recipients at hospitals in Brazil during 1985-2001 had a 0.6\% prevalence of IF (1). During 2007-2009, $\approx 10$ years later, a prospective multicenter study by Nucci et al. reported a 3.7\% IF prevalence among allogeneic HSCT patients and 3.4\% among patients with acute myeloid leukemia (7). That study also found a 1-year cumulative incidence of $5.2 \%$ among those who had received an allogeneic HSCT and 3.8\% among those with acute myeloid leukemia or myelodysplasia (7). A $>10$-fold increase, 
Table 3. Univariate and multivariate Cox regression analyses for 90-day death rate in 50 patients treated for invasive fusariosis, Spain, 2000-2015*

\begin{tabular}{|c|c|c|c|c|}
\hline Characteristic & $\begin{array}{c}\text { Unadjusted hazard ratio } \\
(95 \% \mathrm{Cl})\end{array}$ & $p$ value & $\begin{array}{c}\text { Adjusted hazard ratio } \\
(95 \% \mathrm{Cl})\end{array}$ & $\mathrm{p}$ value \\
\hline \multicolumn{5}{|l|}{ Sex } \\
\hline M & $1.08(0.52-2.25)$ & 0.84 & $0.87(0.41-1.84)$ & 0.87 \\
\hline $\mathrm{F}$ & $0.93(0.45-1.93)$ & 0.84 & & \\
\hline Median age, y (IQR) & $1.00(0.99-1.01)$ & 0.94 & & \\
\hline Charlson index & $1.01(0.86-1.20)$ & 0.87 & $1.16(0.96-1.40)$ & 0.12 \\
\hline \multicolumn{5}{|l|}{ Treatment history (previous $30 \mathrm{~d}$ ) } \\
\hline Antifungal & $1.83(0.81-4.14)$ & 0.15 & & \\
\hline Corticosteroid & $0.69(0.33-1.45)$ & 0.33 & & \\
\hline Neutropenia at onset & $3.16(0.95-10.44)$ & 0.06 & & \\
\hline \multicolumn{5}{|l|}{ Neutropenia at the end of follow-up } \\
\hline Nonneutropenia & Referent & & Referent & \\
\hline Recovery from neutropenia & $1.47(0.38-5.69)$ & 0.58 & $1.91(0.48-7.64)$ & 0.35 \\
\hline Persistent neutropenia & $5.62(1.65-19.11)$ & 0.006 & $9.27(2.43-35.42)$ & 0.001 \\
\hline Hematologic malignancy & $2.44(0.74-8.06)$ & 0.14 & & \\
\hline History of hematopoietic stem cell transplant & $1.39(0.65-2.99)$ & 0.4 & & \\
\hline Fungemia & $0.21(0.03-1.54)$ & 0.13 & & \\
\hline Disseminated disease & $1.90(0.77-4.67)$ & 0.16 & & \\
\hline \multicolumn{5}{|l|}{ Antifungal therapy } \\
\hline Monotherapy with lipid formulation of amphotericin B & $1.95(0.92-4.12)$ & 0.08 & & \\
\hline Monotherapy with azoles & $0.47(0.19-1.15)$ & 0.10 & & \\
\hline Combined therapy & $1.08(0.50-2.33)$ & 0.84 & & \\
\hline
\end{tabular}

from 86 to 1,023 IF cases per 100,000 admissions, occurred among patients with hematologic malignancies at a healthcare center in Brazil from 2000-2005 to 2006-2010 (5). In the United States, a prospective multicenter study conducted by the Transplant-Associated Infection Surveillance Network during 2001-2006 reported a 1-year cumulative incidence of up to $0.3 \%$ of non-Aspergillus mold infections (23). In Italy, a multicenter retrospective study reported a $0.2 \%$ incidence of IF among patients who had received an allogenic HSCT during 1999-2003 (24).

In this cohort in Spain, IF occurred frequently in patients with hematologic conditions and in HSCT recipients. IF occurs almost entirely in markedly immunosuppressed patients who usually are neutropenic; we identified IF in nonneutropenic patients, including patients with a history of solid organ transplants, chronic cardiac or lung disease, or rheumatoid arthritis. These findings are in agreement with Park et al. (9), who studied 37 patients with IF and noted that $54.1 \%$ of IF patients were nonneutropenic, $83.8 \%$ had hematologic malignancies, and $16.2 \%$ had history of solid organ transplantation (9). Thus, nonneutropenia and certain nonhematologic conditions might not be uncommon among IF patients.

Lungs, the bloodstream, and the skin were the organs most frequently affected by IF. Fusarium species produce aleuroconidia, yeastlike structures that can invade the bloodstream and might cause fungemia and metastatic skin lesions (25).

IF is associated with high death rates. This cohort had a 90 -day death rate of $56.9 \%$, similar to rates

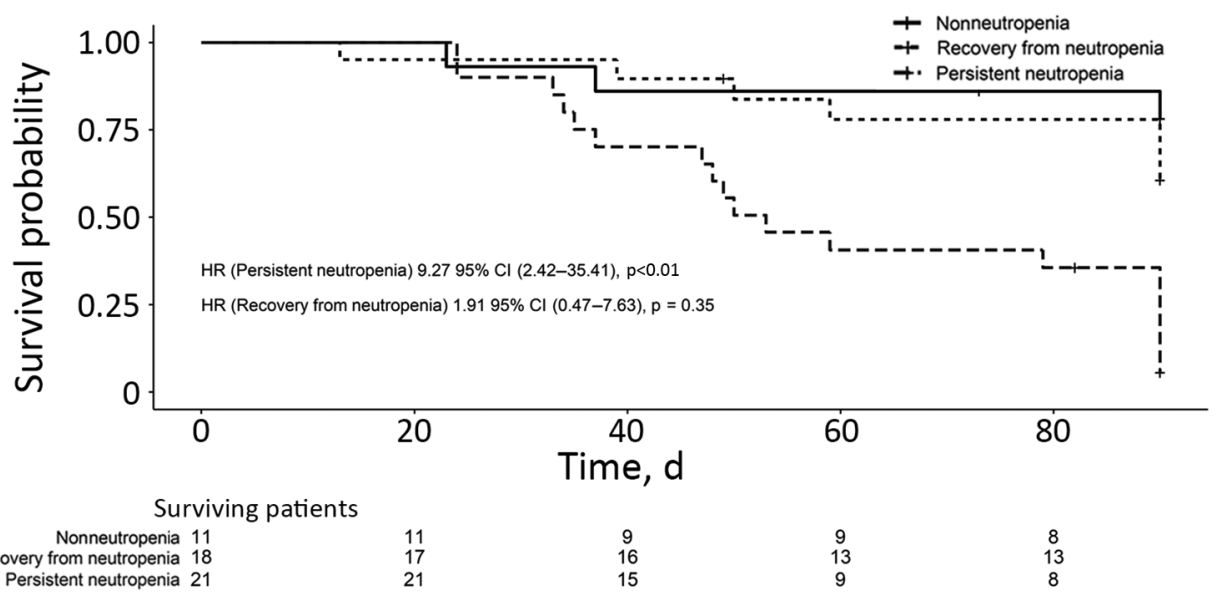

Figure. Adjusted Kaplan-Meier curves obtained from the stratified Cox regression model for 90-day survival in 50 patients treated for invasive fusariosis, Spain, 20002015. HR, hazard ratio. 
noted in other studies $(3,4,12,26)$. The death rates of nonneutropenic and neutropenic patients who recovered from neutropenia were similar $(28.6 \%$ of nonneutropenic patients vs. $38.1 \%$ of neutropenic patients; $\mathrm{p}=0.56$ ), consistent with previous reports that $70 \%$ of IF cases resolve when patients recover from neutropenia (27). In this cohort, patients with persistent neutropenia had a $91.3 \%$ death rate despite antifungal therapy. Persistent neutropenia was the single most predictive prognostic factor in IF, consistent with previous reports (2).

Researchers must identify effective therapeutic strategies to improve the prognosis of IF patients with persistent neutropenia. The treatment practices observed in our study align with guidelines from the European Society of Clinical Microbiology and Infectious Diseases Fungal Infection Study Group and European Confederation of Medical Mycology (13). We examined the effects of different therapeutic regimens on 90 -day death rate. We found no differences in the outcome of patients treated with voriconazole and those receiving a lipid formulation of amphotericin B. Combination therapy also was not associated with outcome, consistent with results from the largest study series conducted so far $(3,12)$. However, these results must be interpreted with caution. Nucci et al. (3) found that patients receiving combination therapy had more severe disease (3), possibly influencing the clinician's decision to administer 2 drugs. In our study, no nonneutropenic patients received combination therapy; further studies should examine the potential benefits of combination therapy in nonneutropenic patients. Finally, because we observed similar clinical responses in patients treated with a lipid formulation of amphotericin B or voriconazole as monotherapies, clinicians might not need to rely on antifungal susceptibility tests to guide treatment. Most clinically relevant Fusarium isolates exhibit high minimal inhibitory concentrations to most antifungals, including azoles, echinocandins, and polyenes (28-30).

Our study is subject to the limitations of retrospective observational studies. For example, the sample size was limited to the cases with available data. Despite being a multicenter study during a 15year period, the sample size might be insufficient to detect significant differences in some groups. Furthermore, clinicians might prescribe a more potent treatment regimen for patients with severe disease, possibly skewing our analysis of treatment outcomes. Although all IF cases in this retrospective study met standard criteria $(16,17)$, we could not determine the causative species in every case.
Thus, the Fusarium species was only reported when determined by genomic sequencing or MALDITOF mass spectrometry, because these techniques have high agreement rates (89.8\%-97.0\%) (31-33). All remaining IF cases in our series were reported as Fusarium spp. We also were not able to culture all Fusarium isolates because of a lack of specimens, especially for cases occurring before 2010 in hospitals in which Fusarium isolates were not collected as part of routine procedures

In conclusion, our data show that IF is an emerging infection in Spain. We report an increase in the incidence of IF among nonneutropenic patients, including those with hematologic conditions and other concurrent conditions, such as chronic cardiac or lung diseases, rheumatoid arthritis, or history of solid organ transplants. Our results support previous studies reporting that IF survival is critically dependent on resolution of neutropenia.

Members of the Spanish Fusariosis Study Group include: Cesar Vargas Ayala, Paula Pescador Martin, and Laura Alonso Acero (Hospital Universitario La Paz, Madrid, Spain); Leire López-Soria, Laura Guio-Carrión, and Roberto Céspedes (Hospital Universitario Cruces, Bilbao, Spain); M. Carmen Martínez Jiménez, Maricela Valerio, and Jesús Guinea (Hospital General Universitario Gregorio Marañón, Madrid, Spain); Elisa Ibáñez Martínez, Sandra CuellarTovar, and Jaime Sanz-Caballer (Hospital Universitario y Politécnico La Fe, Valencia, Spain); Lissette Costilla (Hospital Universitario Miguel Servet, Zaragoza, Spain); Maria Pía Roiz-Mesones (Hospital Universitario Marqués de Valdecilla, Santander, Spain); Cristina Gil-Cortés and Carmen Botella-Prieto (Hospital General Universitario de Alicante, Alicante, Spain); Iballa Horcajada-Herrera (Hospital Universitario de Gran Canaria Doctor Negrín, Las Palmas de Gran Canaria, Spain); Juan José Castón, Alejandra M. Natera, and Ipek Guler (Maimonides Biomedical Research Institute of Cordoba, Reina Sofia University Hospital, University of Cordoba, Cordoba, Spain); Pilar Martín-Dávila and Elia Gómez-García de la Pedrosa (Hospital Universitario Ramón y Cajal, Madrid); Eduardo Aznar-Oroval and Agustín Iranzo (Fundación Instituto Valenciano de Oncología, Valencia); Josefina Ayats and Carlota Gudiol (Hospital Universitario de Bellvitge, Barcelona, Spain); Ana Pérez-Ayala (Hospital Universitario 12 de Octubre, Madrid); Cristina Lopez-Querol and Alicia Mulero Serralta (Hospital de la Santa Creu i Sant Pau de Barcelona, Barcelona); Estrella Martin-Mazuelos (Hospital Universitario Virgen de Valme, Seville, Spain); Manuel Rodríguez-Iglesias (Hospital Universitario Puerta del Mar, Cádiz, Spain); and M. Teresa Martin-Gómez (Hospital Universitari Vall d'Hebrón, Barcelona). 
This work was supported by Plan Nacional de I+D+I 2013-2016 and Instituto de Salud Carlos III, Subdirección General de Redes y Centros de Investigación Cooperativa, Ministerio de Ciencia, Innovación y Universidades, Spanish Network for Research in Infectious Diseases (REIPI RD16/0016/0008; RD16/CIII/0004/0003), cofinanced by European Development Regional Fund "A way to achieve Europe," Operative Program Intelligent Growth 2014-2020. M.F.R. holds a "Miguel Servet" research contract (CP18/00073) from Instituto de Salud Carlos III, Madrid.

\section{About the Author}

Dr. Pérez-Nadales is a research fellow at the Infectious Diseases Group at Maimonides Biomedical Research Institute of Córdoba, Spain. Her research interests include clinical and molecular epidemiology of invasive fungal infections and infections caused by multidrugresistant bacteria.

\section{References}

1. Nucci M, Marr KA, Queiroz-Telles F, Martins CA, Trabasso P, Costa S, et al. Fusarium infection in hematopoietic stem cell transplant recipients. Clin Infect Dis. 2004;38:1237-42. https:/ / doi.org/10.1086/383319

2. Nucci M, Anaissie EJ, Queiroz-Telles F, Martins CA, Trabasso P, Solza C, et al. Outcome predictors of 84 patients with hematologic malignancies and Fusarium infection. Cancer. 2003;98:315-9. https://doi.org/10.1002/cncr.11510

3. Nucci M, Marr KA, Vehreschild MJGT, de Souza CA, Velasco E, Cappellano P, et al. Improvement in the outcome of invasive fusariosis in the last decade. Clin Microbiol Infect. 2014;20:580-5. https:/ / doi.org/10.1111/1469-0691.12409

4. Horn DL, Freifeld AG, Schuster MG, Azie NE, Franks B, Kauffman CA. Treatment and outcomes of invasive fusariosis: review of 65 cases from the PATH Alliance registry. Mycoses. 2014;57:652-8. https://doi.org/10.1111/myc.12212

5. Nucci M, Varon AG, Garnica M, Akiti T, Barreiros G, Trope BM, et al. Increased incidence of invasive fusariosis with cutaneous portal of entry, Brazil. Emerg Infect Dis. 2013;19:1567-72. https://doi.org/10.3201/eid1910.120847

6. Nucci M, Anaissie E. Cutaneous infection by Fusarium species in healthy and immunocompromised hosts: implications for diagnosis and management. Clin Infect Dis. 2002;35:909-20. https:// doi.org/10.1086/342328

7. Nucci M, Garnica M, Gloria AB, Lehugeur DS, Dias VCH, Palma LC, et al. Invasive fungal diseases in haematopoietic cell transplant recipients and in patients with acute myeloid leukaemia or myelodysplasia in Brazil. Clin Microbiol Infect. 2013;19:745-51. https:// doi.org/10.1111/1469-0691.12002

8. Girmenia C, Pagano L, Corvatta L, Mele L, del Favero A, Martino P. The epidemiology of fusariosis in patients with haematological diseases. Gimema Infection Programme. Br J Haematol. 2000;111:272-6.

9. Park BJ, Pappas PG, Wannemuehler KA, Alexander BD, Anaissie EJ, Andes DR, et al. Invasive non-Aspergillus mold infections in transplant recipients, United States, 2001-2006. Emerg Infect Dis. 2011;17:1855-64. https:/ / doi.org/10.3201/ eid1710.110087

10. Hennequin C, Lavarde V, Poirot JL, Rabodonirina M, Datry A, Aractingi S, et al. Invasive Fusarium infections: a retrospective survey of 31 cases. J Med Vet Mycol. 1997;35:107-14. https://doi.org/10.1080/02681219780000991

11. Pagano L, Caira M, Candoni A, Offidani M, Fianchi L, Martino B, et al. The epidemiology of fungal infections in patients with hematologic malignancies: the SEIFEM-2004 study. Haematologica. 2006;91:1068-75.

12. Lortholary O, Obenga G, Biswas P, Caillot D, Chachaty E, Bienvenu A-L, et al.; French Mycoses Study Group. International retrospective analysis of 73 cases of invasive fusariosis treated with voriconazole. Antimicrob Agents Chemother. 2010;54:4446-50. https:/ / doi.org/10.1128/ AAC.00286-10

13. Tortorano AM, Prigitano A, Esposto MC, Arsic Arsenijevic V, Kolarovic J, Ivanovic D, et al.; ECMM Working Group. European Confederation of Medical Mycology (ECMM) epidemiological survey on invasive infections due to Fusarium species in Europe. Eur J Clin Microbiol Infect Dis. 2014;33:1623-30. https:/ /doi.org/10.1007/s10096-014-2111-1

14. Tortorano AM, Prigitano A, Dho G, Esposto MC, Gianni C, Grancini A, et al. Species distribution and in vitro antifungal susceptibility patterns of 75 clinical isolates of Fusarium spp. from northern Italy. Antimicrob Agents Chemother. 2008;52:2683-5. https://doi.org/10.1128/AAC.00272-08

15. Dalyan Cilo B, Al-Hatmi AMS, Seyedmousavi S, Rijs AJMM, Verweij PE, Ener B, et al. Emergence of fusarioses in a university hospital in Turkey during a 20-year period. Eur J Clin Microbiol Infect Dis. 2015;34:1683-91. https://doi.org/10.1007/s10096-015-2405-y

16. Donnelly JP, Chen SC, Kauffman CA, Steinbach WJ, Baddley JW, Verweij PE, et al. Revision and Update of the Consensus Definitions of Invasive Fungal Disease From the European Organization for Research and Treatment of Cancer and the Mycoses Study Group Education and Research Consortium. Clin Infect Dis. 2020;71:1367-76. https://doi.org/10.1093/cid/ciz1008

17. De Pauw B, Walsh TJ, Donnelly JP, Stevens DA, Edwards JE, Calandra T, et al.; European Organization for Research and Treatment of Cancer/Invasive Fungal Infections Cooperative Group; National Institute of Allergy and Infectious Diseases Mycoses Study Group (EORTC/MSG) Consensus Group. Revised definitions of invasive fungal disease from the European Organization for Research and Treatment of Cancer/Invasive Fungal Infections Cooperative Group and the National Institute of Allergy and Infectious Diseases Mycoses Study Group (EORTC/MSG) Consensus Group. Clin Infect Dis. 2008;46:1813-21. https:/ / doi.org/ 10.1086/588660

18. Geiser DM, del Mar Jiménez-Gasco M, Kang S, Makalowska I, Veeraraghavan N, Ward TJ, et al. FUSARIUM-ID v. 1.0: A DNA sequence database for identifying Fusarium. Eur J Plant Pathol. 2004;110:473-9. https:// doi.org/10.1023/B:EJPP.0000032386.75915.a0

19. Holden D. DNA mini prep method for Aspergillus fumigatus (and other filamentous fungi). In: Maresca B, Kobayashi G, editors. Molecular biology of pathogenic fungi, a laboratory manual. New York: Telos Press; 1994. p. 3-4.

20. Alastruey-Izquierdo A, Alcazar-Fuoli L, Rivero-Menéndez O, Ayats J, Castro C, García-Rodríguez J, et al. Molecular identification and susceptibility testing of molds isolated in a prospective surveillance of triazole resistance in Spain (FILPOP2 Study). Antimicrob Agents Chemother. 2018;62. AAC.00358-18.

21. Bernal-Martínez L, Buitrago MJ, Castelli MV, Rodríguez-Tudela JL, Cuenca-Estrella M. Detection of invasive infection caused by Fusarium solani and nonFusarium solani species using a duplex quantitative PCR- 
based assay in a murine model of fusariosis. Med Mycol. 2012;50:270-5. https:// doi.org/10.3109/13693786.2011.604047

22. Charlson M, Szatrowski TP, Peterson J, Gold J. Validation of a combined comorbidity index. J Clin Epidemiol. 1994; 47:1245-51. https://doi.org/10.1016/0895-4356(94)90129-5

23. Kontoyiannis DP, Marr KA, Park BJ, Alexander BD, Anaissie EJ, Walsh TJ, et al. Prospective surveillance for invasive fungal infections in hematopoietic stem cell transplant recipients, 2001-2006: overview of the Transplant-Associated Infection Surveillance Network (TRANSNET) Database. Clin Infect Dis. 2010;50:1091-100. https://doi.org/10.1086/651263

24. Pagano L, Caira M, Nosari A, Van Lint MT, Candoni A, Offidani $\mathrm{M}$, et al. Fungal infections in recipients of hematopoietic stem cell transplants: results of the SEIFEM B-2004 study - Sorveglianza Epidemiologica Infezioni Fungine Nelle Emopatie Maligne. Clin Infect Dis. 2007;45:1161-70. https:// doi.org/10.1086/522189

25. Anaissie E, Grazziutti M, Nucci M. Invasive fungal infections in cancer patients. In: Anaissie E, McGinnis M, Pfaller M, editors. Clinical mycology. 2nd ed. Amsterdam: Elsevier; 2009. p. 431-71.

26. Campo M, Lewis RE, Kontoyiannis DP. Invasive fusariosis in patients with hematologic malignancies at a cancer center: 1998-2009. J Infect. 2010;60:331-7. https:/ / doi.org/10.1016/ j.jinf.2010.01.010

27. Lionakis MS, Kontoyiannis DP. Fusarium infections in critically ill patients. Semin Respir Crit Care Med. 2004;25:159-69. https://doi.org/10.1055/s-2004-824900

28. Al-Hatmi AMS, van Diepeningen AD, Curfs-Breuker I, de Hoog GS, Meis JF. Specific antifungal susceptibility profiles of opportunists in the Fusarium fujikuroi complex. J Antimicrob Chemother. 2015;70:1068-71. bMed

29. Alastruey-Izquierdo A, Cuenca-Estrella M, Monzón A, Mellado E, Rodríguez-Tudela JL. Antifungal susceptibility profile of clinical Fusarium spp. isolates identified by molecular methods. J Antimicrob Chemother. 2008;61:805-9. https://doi.org/10.1093/jac/dkn022

30. Al-Hatmi AMS, Normand A-C, Ranque S, Piarroux R, de Hoog GS, Meletiadis J, et al. Comparative evaluation of Etest, EUCAST, and CLSI Methods for amphotericin B, voriconazole, and posaconazole against clinically relevant Fusarium species. Antimicrob Agents Chemother. 2016;61:e01671-16.

31. Marinach-Patrice C, Lethuillier A, Marly A, Brossas JY, Gené J, Symoens F, et al. Use of mass spectrometry to identify clinical Fusarium isolates. Clin Microbiol Infect. 2009; 15:634-42. https://doi.org/10.1111/j.1469-0691. 2009.02758.x

32. Triest D, Stubbe D, De Cremer K, Piérard D, Normand AC, Piarroux R, et al. Use of matrix-assisted laser desorption ionization-time of flight mass spectrometry for identification of molds of the Fusarium genus. J Clin Microbiol. 2015;53:465-76. https:/ / doi.org/10.1128/ JCM.02213-14

33. Paziani MH, Tonani Carvalho L, Melhem MSC, Almeida MTG, Nadaletto Bonifácio da Silva ME, Martinez R, et al. First comprehensive report of clinical Fusarium strains isolated in the state of Sao Paulo (Brazil) and identified by MALDI-TOF MS and molecular biology. Microorganisms. 2019;8:66. https://doi.org/10.3390/ microorganisms 8010066

Address for correspondence: Julián Torre-Cisneros, Clinical Unit of Infectious Diseases, Reina Sofía University Hospital, Maimonides Biomedical Research Institute of Córdoba (IMIBIC), University of Córdoba, Córdoba, Spain; e-mail: julian.torre.sspa@juntadeandalucia.es 